\title{
Secondhand Smoke Exposure, Depression Symptoms, and Suicidal Ideation in Adults
}

\author{
Soo Young Kim \\ Department of Family Medicine, Kangdong Sacred Heart Hospital, Hallym University College of Medicine, Seoul, Korea
}

Secondhand smoke, also called 'environmental tobacco smoke', is a combination of smoke emitted from the burning end of a cigarette and smoke exhaled by a smoker. Many epidemiological studies have suggested that secondhand smoke exposure (passive smoking) is related to an increased prevalence of cardiovascular diseases and several cancers including lung, breast, and pancreatic cancer. Recent research has revealed that secondhand smoke exposure may contribute to mental health issues in adults. ${ }^{1)}$ Some studies have suggested that secondhand smoke exposure is associated with depression symptoms. However, there is a lack of studies investigating the relationship between secondhand smoke exposure and suicidal ideation.

In the present issue, Gim et al. ${ }^{2)}$ evaluated the effect of secondhand smoke on depressive symptoms and suicidal ideation in Korean adults. The authors obtained the data from 2010-2012 Korea National Health and Nutrition Examination Survey, which consisted of 13,006 participants who were exposed to secondhand smoke in the office or at home, and 3,037 non-exposed participants. The authors found that the exposure to secondhand smoke was associated with an increase in depressive symptoms and suicidal ideation among adults.

These results are similar to the findings from previous research that secondhand smoke may increase the risk of depressive symptoms. ${ }^{3,4)}$ However, the unique finding of this study is that secondhand smoke may be associated with suicidal ideas.

Following the enactment of the National Health Promotion Act in Korea in 1995, many public areas such as government buildings, large restaurants, public parks, streets, and bus stations were designated as non-smoking areas. However, according to the 2006 report of the Surgeon General, ${ }^{5)}$ eliminat- ing smoking in indoor spaces fully protects nonsmokers from exposure to secondhand smoke. Separating smokers from nonsmokers, cleaning the air, and ventilating the buildings cannot eliminate exposure of nonsmokers to secondhand smoke. A more powerful enactment may be needed.

\section{CONFLICT OF INTEREST}

No potential conflict of interest relevant to this article was reported.

\section{REFERENCES}

1. Zeng YN, Li YM. Secondhand smoke exposure and mental health in adults: a meta-analysis of cross-sectional studies. Soc Psychiatry Psychiatr Epidemiol 2015 Dec 10 [Epub]. http://dx.doi.org/ 10.1007/s00127-015-1164-5.

2. Gim W, Yoo JH, Shin JY, Goo AJ. Relationship between secondhand smoking with depressive symptom and suicidal ideation in Korean non-smoker adults: the Korean National Health and Nutrition Examination Survey 2010-2012. Korean J Fam Med 2016;37:97-104.

3. Jung SJ, Shin A, Kang D. Active smoking and exposure to secondhand smoke and their relationship to depressive symptoms in the Korea national health and nutrition examination survey (KNHANES). BMC Public Health 2015;15:1053.

4. Lee KJ. Current smoking and secondhand smoke exposure and depression among Korean adolescents: analysis of a national cross-sectional survey. BMJ Open 2014;4:e003734.

5. United States Public Health Service, Department of Health and Human Services. The health consequences of involuntary exposure to tobacco smoke: a report of the Surgeon General. Rockville (MD): US Department of Health and Human Services; 2006. 